\title{
The reverse order law for EP modular operators
}

\author{
Javad Farokhi-Ostad ${ }^{a, *}$, Mehdi Mohammadzadeh Karizaki ${ }^{\mathrm{b}}$ \\ a Department of Basic Sciences, Birjand University of Technology, Birjand, Iran. \\ ${ }^{b}$ University of Torbat Heydarieh, Torbat Heydariyeh, Iran.
}

\begin{abstract}
In this paper, we present new conditions that reverse order law holds for EP modular operators. (C)2016 All rights reserved.

Keywords: EP operator, reverse order law, Moore-Penrose inverse, Hilbert $C^{*}$-module, closed range.

$2010 M S C: 47 \mathrm{~A} 05,46 \mathrm{~L} 08,15 \mathrm{~A} 09$.
\end{abstract}

\section{Introduction and preliminaries}

It is a classical result of Greville [8, that $(T S)^{\dagger}=S^{\dagger} T^{\dagger}$ if and only if $\operatorname{ran}\left(T^{*} T S\right) \subset \operatorname{ran}(S)$ and $\operatorname{ran}\left(S S^{*} T^{*}\right) \subset \operatorname{ran}\left(T^{*}\right)$, in the case when $T$ and $S$ are complex (possibly rectangular) matrices. This result is extended for bounded linear operators on Hilbert spaces, by Bouldin [2, 3], and Izumino [9]. Then, in [5], the reverse order law for the Moore-Penrose inverse is obtained as a consequence of some set equalities. Recently, Sharifi [13] and Mohammadzadeh Karizaki [11, 12] studied Moore -Penrose inverse of product of the operators with closed range in Hilbert $C^{*}$-modules.

In this paper, we state new conditions that reverse order law for the Moore-Penrose inverse holds. A bounded linear operator $T$ with closed range on a complex Hilbert space $H$ is called an EP operator if $T$ and $T^{*}$ have the same range. Djordjević [4] gave necessary and sufficient conditions for a product of two EP operators with closed ranges to be an EP operator with a closed range. In addition, we state new conditions that if $T, S \in \mathcal{L}(\mathcal{X})$ are EP operators with closed ranges and $T S=S T^{*}=S^{*} T$, then $T S$ has closed range and $(T S)^{\dagger}=S^{\dagger} T^{\dagger}$.

\footnotetext{
*Corresponding author

Email addresses: javadfarrokhi90@gmail.com, j.farrokhi@birjandut.ac.ir (Javad Farokhi-Ostad), mohammadzadehkarizaki@gmail.com (Mehdi Mohammadzadeh Karizaki)
} 
Hilbert $C^{*}$-modules are objects like Hilbert spaces, except that the inner product takes its values in a $C^{*}$-algebra, instead of being complex-valued. Throughout the paper, $\mathcal{A}$ is a $C^{*}$-algebra (not necessarily unital). A (right) pre-Hilbert module over a $C^{*}$-algebra $\mathcal{A}$ is a complex linear space $\mathcal{X}$, which is an algebraic right $\mathcal{A}$-module and $\lambda(x a)=(\lambda x) a=x(\lambda a)$ equipped with an $\mathcal{A}$-valued inner product $\langle.,\rangle:. \mathcal{X} \times \mathcal{X} \rightarrow \mathcal{A}$ satisfying,

(i) $\langle x, x\rangle \geq 0$, and $\langle x, x\rangle=0$ iff $x=0$,

(ii) $\langle x, y+\lambda z\rangle=\langle x, y\rangle+\lambda\langle x, z\rangle$,

(iii) $\langle x, y a\rangle=\langle x, y\rangle a$,

(iv) $\langle y, x\rangle=\langle x, y\rangle^{*}$

for each $x, y, z \in \mathcal{X}, \lambda \in \mathbb{C}, a \in \mathcal{A}$. A pre-Hilbert $\mathcal{A}$-module $\mathcal{X}$ is called a Hilbert $\mathcal{A}$-module if it is complete with respect to the norm $\|x\|=\|\langle x, x\rangle\|^{\frac{1}{2}}$. Left Hilbert $\mathcal{A}$-modules are defined in a similar way. For example, every $C^{*}$-algebra $\mathcal{A}$ is a Hilbert $\mathcal{A}$-module with respect to inner product $\langle x, y\rangle=x^{*} y$, and every inner product space is a left Hilbert $\mathbb{C}$-module.

Suppose that $\mathcal{X}$ and $\mathcal{Y}$ are Hilbert $\mathcal{A}$-modules, then, $\mathcal{L}(\mathcal{X}, \mathcal{Y})$ is the set of all maps $T: \mathcal{X} \rightarrow \mathcal{Y}$ for which there is a map $T^{*}: \mathcal{Y} \rightarrow \mathcal{X}$, the so-called adjoint of $T$ such that $\langle T x, y\rangle=\left\langle x, T^{*} y\right\rangle$ for each $x \in \mathcal{X}, y \in \mathcal{Y}$. It is known that any element $T$ of $\mathcal{L}(\mathcal{X}, \mathcal{Y})$ must be a bounded linear operator, which is also $\mathcal{A}$-linear in the sense that $T(x a)=(T x) a$ for $x \in \mathcal{X}$ and $a \in \mathcal{A}$ [10, Page 8]. We use the notations $\mathcal{L}(\mathcal{X})$ in place of $\mathcal{L}(\mathcal{X}, \mathcal{X})$, and $\operatorname{ker}(\cdot)$ and $\operatorname{ran}(\cdot)$ for the kernel and the range of operators, respectively. The identity operator on $\mathcal{X}$ is denoted by $1_{\mathcal{X}}$ or 1 if there is no ambiguity.

Suppose that $\mathcal{X}$ is a Hilbert $\mathcal{A}$-module and $\mathcal{Y}$ is a closed submodule of $\mathcal{X}$. We say that $\mathcal{Y}$ is orthogonally complemented if $\mathcal{X}=\mathcal{Y} \oplus \mathcal{Y}^{\perp}$, where $\mathcal{Y}^{\perp}:=\{y \in \mathcal{X}:\langle x, y\rangle=0$ for all $x \in \mathcal{Y}\}$ denotes the orthogonal complement of $\mathcal{Y}$ in $\mathcal{X}$. The reader is referred to [6, 17, 10] and the references cited therein for more details.

Throughout this paper, $\mathcal{X}$ and $\mathcal{Y}$ are Hilbert $\mathcal{A}$-modules. Recall that a closed submodule in a Hilbert module is not necessarily orthogonally complemented, however, Lance [10] proved that certain submodules are orthogonally complemented as follows:

Theorem $1.1([10])$. Suppose that $T \in \mathcal{L}(\mathcal{X}, \mathcal{Y})$ has closed range. Then

- $\operatorname{ker}(T)$ is orthogonally complemented in $\mathcal{X}$, with complement $\operatorname{ran}\left(T^{*}\right)$;

- $\operatorname{ran}(T)$ is orthogonally complemented in $\mathcal{Y}$, with complement $\operatorname{ker}\left(T^{*}\right)$;

- The map $T^{*} \in \mathcal{L}(\mathcal{Y}, \mathcal{X})$ has closed range.

$\mathrm{Xu}$ and Sheng [16] have shown that a bounded adjointable operator between two Hilbert $C^{*}$ modules admits a bounded Moore-Penrose inverse if and only if the operator has closed range.

Definition 1.2. Let $T \in \mathcal{L}(\mathcal{X}, \mathcal{Y})$. The Moore-Penrose inverse $T^{\dagger}$ of $T$ (if it exists) is an element in $X \in L(\mathcal{Y}, \mathcal{X})$ which satisfies:

1. $T X T=T$

2. $X T X=X$;

3. $(T X)^{*}=T X$;

4. $(X T)^{*}=X T$.

If $\theta \subseteq\{1,2,3,4\}$, and $X$ satisfies the equations (ii) for all $i \in \theta$, then $X$ is a $\theta$-inverse of $T$. The set of all $\theta$-inverses of $T$ is denoted by $T\{\theta\}$. If $\operatorname{ran}(T)$ is closed, then $T\{1,2,3,4\}=\left\{T^{\dagger}\right\}$. An operator $X$ is an inner inverse of $T$ if $X \in T\{1\}$. In this case, $T$ is inner invertible, or relatively regular. It is well-known that $T$ is inner invertible if and only if $\operatorname{ran}(T)$ is closed. 
Motivated by these conditions, $T^{\dagger}$ is unique and $T^{\dagger} T$ and $T T^{\dagger}$ are orthogonal projections, in the sense that they are self-adjoint idempotent operators. Clearly, $T$ is Moore-Penrose invertible if and only if $T^{*}$ is Moore-Penrose invertible, and in this case $\left(T^{*}\right)^{\dagger}=\left(T^{\dagger}\right)^{*}$.

By Definition 1.2, we have

$$
\begin{array}{rlrl}
\operatorname{ran}(T) & =\operatorname{ran}\left(T T^{\dagger}\right), & & \operatorname{ran}\left(T^{\dagger}\right)=\operatorname{ran}\left(T^{\dagger} T\right)=\operatorname{ran}\left(T^{*}\right), \\
\operatorname{ker}(T)=\operatorname{ker}\left(T^{\dagger} T\right), & & \operatorname{ker}\left(T^{\dagger}\right)=\operatorname{ker}\left(T T^{\dagger}\right)=\operatorname{ker}\left(T^{*}\right),
\end{array}
$$

and by Theorem 1.1 , we obtain

$$
\begin{aligned}
& \mathcal{X}=\operatorname{ker}(T) \oplus \operatorname{ran}\left(T^{\dagger}\right)=\operatorname{ker}\left(T^{\dagger} T\right) \oplus \operatorname{ran}\left(T^{\dagger} T\right) \\
& \mathcal{Y}=\operatorname{ker}\left(T^{\dagger}\right) \oplus \operatorname{ran}(T)=\operatorname{ker}\left(T T^{\dagger}\right) \oplus \operatorname{ran}\left(T T^{\dagger}\right)
\end{aligned}
$$

A matrix form of a bounded adjointable operator $T \in \mathcal{L}(\mathcal{X}, \mathcal{Y})$ can be induced by some natural decompositions of Hilbert $C^{*}$-modules. Indeed, if $\mathcal{M}$ and $\mathcal{N}$ are closed orthogonally complemented submodules of $\mathcal{X}$ and $\mathcal{Y}$, respectively, and $\mathcal{X}=\mathcal{M} \oplus \mathcal{M}^{\perp}, \mathcal{Y}=\mathcal{N} \oplus \mathcal{N}^{\perp}$, then $T$ can be written as the following $2 \times 2$ matrix

$$
T=\left[\begin{array}{ll}
T_{1} & T_{2} \\
T_{3} & T_{4}
\end{array}\right]
$$

where, $T_{1} \in \mathcal{L}(\mathcal{M}, \mathcal{N}), T_{2} \in \mathcal{L}\left(\mathcal{M}^{\perp}, \mathcal{N}\right), T_{3} \in \mathcal{L}\left(\mathcal{M}, \mathcal{N}^{\perp}\right)$ and $T_{4} \in \mathcal{L}\left(\mathcal{M}^{\perp}, \mathcal{N}^{\perp}\right)$. Note that $P_{\mathcal{M}}$ denotes the projection corresponding to $\mathcal{M}$.

In fact $T_{1}=P_{\mathcal{N}} T P_{\mathcal{M}}, T_{2}=P_{\mathcal{N}} T\left(1-P_{\mathcal{M}}\right), T_{3}=\left(1-P_{\mathcal{N}}\right) T P_{\mathcal{M}}, T_{4}=\left(1-P_{\mathcal{N}}\right) T\left(1-P_{\mathcal{M}}\right)$.

Definition $1.3([14])$. Let $\mathcal{X}$ be a Hilbert $\mathcal{A}$-modules. An operator $T \in \mathcal{L}(\mathcal{X})$ is called EP if $\operatorname{ran}(T)$ and $\operatorname{ran}\left(T^{*}\right)$ have the same closure.

Lemma 1.4 ([14]). Let $\mathcal{X}$ be a Hilbert $\mathcal{A}$-module and $T \in \mathcal{L}(\mathcal{X})$ with closed range. Then $T$ is $E P$ if and only if it is of the matrix form

$$
T=\left[\begin{array}{cc}
T_{1} & 0 \\
0 & 0
\end{array}\right]:\left[\begin{array}{c}
\operatorname{ran}(T) \\
\operatorname{ker}(T)
\end{array}\right] \rightarrow\left[\begin{array}{l}
\operatorname{ran}(T) \\
\operatorname{ker}(T)
\end{array}\right]
$$

for some invertible operator $T_{1} \in \mathcal{L}(\operatorname{ran}(T), \operatorname{ran}(T))$.

\section{The reverse order law}

In this section, we state some new conditions that reverse order law holds for EP modular operators.

Theorem 2.1. Suppose that $\mathcal{X}$ is a Hilbert $\mathcal{A}$-module and $T, S \in \mathcal{L}(\mathcal{X})$ are EP operators with closed ranges and $T S=S T^{*}$. Then $T S$ has closed range and $(T S)^{\dagger} T S=S^{\dagger} T^{\dagger} T S$.

Moreover, if $P_{\operatorname{ran}(\mathrm{S})} T=P_{\operatorname{ran}(\mathrm{S})} T P_{\operatorname{ran}(\mathrm{S})}$, then

(i) $T S(T S)^{\dagger}=T S S^{\dagger} T^{\dagger}$,

(ii) $(T S)^{\dagger}=S^{\dagger} T^{\dagger}$.

Proof. Since $T$ is EP operator with closed range, then by Lemma 1.4, operators $S$ and $T$ have the following matrix representations

$$
T=\left[\begin{array}{cc}
T_{1} & 0 \\
0 & 0
\end{array}\right]:\left[\begin{array}{c}
\operatorname{ran}(T) \\
\operatorname{ker}(T)
\end{array}\right] \rightarrow\left[\begin{array}{c}
\operatorname{ran}(T) \\
\operatorname{ker}(T)
\end{array}\right],
$$




$$
S=\left[\begin{array}{ll}
S_{1} & S_{2} \\
S_{3} & S_{4}
\end{array}\right]:\left[\begin{array}{c}
\operatorname{ran}(T) \\
\operatorname{ker}(T)
\end{array}\right] \rightarrow\left[\begin{array}{l}
\operatorname{ran}(T) \\
\operatorname{ker}(T)
\end{array}\right]
$$

Since $T S=S T^{*}$, then

$$
\left[\begin{array}{cc}
T_{1} & 0 \\
0 & 0
\end{array}\right]\left[\begin{array}{ll}
S_{1} & S_{2} \\
S_{3} & S_{4}
\end{array}\right]=\left[\begin{array}{cc}
S_{1} & S_{2} \\
S_{3} & S_{4}
\end{array}\right]\left[\begin{array}{cc}
T_{1}^{*} & 0 \\
0 & 0
\end{array}\right]
$$

or equivalently,

$$
\left[\begin{array}{cc}
T_{1} S_{1} & T_{1} S_{2} \\
0 & 0
\end{array}\right]=\left[\begin{array}{ll}
S_{1} T_{1} & 0 \\
S_{3} T_{1} & 0
\end{array}\right]
$$

Equation (2.3) shows that $T_{1} S_{2}=S_{3} T_{1}=0$, by invertibility of $T_{1}$ we conclude that $S_{2}=S_{3}=0$. Since $\operatorname{ran}(S)$ is closed, then $\operatorname{ran}\left(S_{1}\right)$ and $\operatorname{ran}\left(S_{4}\right)$ are closed. We let $\left\{y_{n}=z_{n} \oplus x_{n}\right\}$ be a sequence chosen in $\operatorname{ran}(T) \oplus \operatorname{ker}(T),\left\{z_{n}\right\},\left\{x_{n}\right\}$ be sequences chosen in $\operatorname{ran}(T)$ and $\operatorname{ker}(T)$, respectively, such that $S\left(z_{n} \oplus x_{n}\right) \rightarrow y$. Since $\operatorname{ran}(\mathrm{S})$ is assumed to be closed, then $y \in \operatorname{ran}(\mathrm{S})$. On the other hand, $y=z+x$ for some $z \in \operatorname{ran}(T)$ and $x \in \operatorname{ker}(T)$. By direct sum property, $S\left(z_{n} \oplus x_{n}\right)=\left(S_{1} \oplus S_{4}\right)\left(z_{n} \oplus x_{n}\right)=$ $S_{1}\left(z_{n}\right) \oplus S_{4}\left(x_{n}\right) \rightarrow z+x$, that is $S_{1}\left(z_{n}\right) \rightarrow z$ and $S_{4}\left(x_{n}\right) \rightarrow x$. Since $S=S_{1} \oplus S_{4}$, then $z \in \operatorname{ran}\left(S_{1}\right)$ and $x \in \operatorname{ran}\left(S_{4}\right)$. This is implies that $\operatorname{ran}\left(S_{1}\right)$ and $\operatorname{ran}\left(S_{4}\right)$ are closed.

Therefore, obviously $S^{\dagger}=\left[\begin{array}{cc}S_{1}^{\dagger} & 0 \\ 0 & S_{4}^{\dagger}\end{array}\right]$ is Moore-Penrose inverse of $S=\left[\begin{array}{cc}S_{1} & 0 \\ 0 & S_{4}\end{array}\right]$. Hence, we have

$$
\begin{aligned}
T S & =\left[\begin{array}{cc}
T_{1} & 0 \\
0 & 0
\end{array}\right]\left[\begin{array}{cc}
S_{1} & 0 \\
0 & S_{4}
\end{array}\right]=\left[\begin{array}{cc}
T_{1} S_{1} & 0 \\
0 & 0
\end{array}\right], \\
S^{\dagger} T^{\dagger} & =\left[\begin{array}{cc}
S_{1}^{\dagger} & 0 \\
0 & S_{4}^{\dagger}
\end{array}\right]\left[\begin{array}{cc}
T_{1}^{-1} & 0 \\
0 & 0
\end{array}\right]=\left[\begin{array}{cc}
S_{1}^{\dagger} T_{1}^{-1} & 0 \\
0 & 0
\end{array}\right] .
\end{aligned}
$$

Since

$$
\begin{aligned}
T S S^{\dagger} T^{\dagger} T S & =\left[\begin{array}{cc}
\left(T_{1} S_{1}\right) S_{1}^{\dagger} T_{1}^{-1}\left(T_{1} S_{1}\right) & 0 \\
0 & 0
\end{array}\right]=\left[\begin{array}{cc}
T_{1} S_{1} & 0 \\
0 & 0
\end{array}\right], \\
S^{\dagger} T^{\dagger} T S S^{\dagger} T^{\dagger} & =\left[\begin{array}{cc}
S_{1}^{\dagger} T^{-1} & 0 \\
0 & 0
\end{array}\right]\left[\begin{array}{cc}
T_{1} S_{1} & 0 \\
0 & 0
\end{array}\right]\left[\begin{array}{cc}
S_{1}^{\dagger} T^{-1} & 0 \\
0 & 0
\end{array}\right]=\left[\begin{array}{cc}
S_{1}^{\dagger} T_{1}^{-1} & 0 \\
0 & 0
\end{array}\right], \\
\left(S^{\dagger} T^{\dagger} T S\right)^{*} & =\left[\begin{array}{cc}
S_{1}^{\dagger} T^{-1} & 0 \\
0 & 0
\end{array}\right]\left[\begin{array}{cc}
T_{1} S_{1} & 0 \\
0 & 0
\end{array}\right]=\left[\begin{array}{cc}
S_{1}^{\dagger} S_{1} & 0 \\
0 & 0
\end{array}\right]=S^{\dagger} T^{\dagger} T S .
\end{aligned}
$$

Then $S^{\dagger} T^{\dagger} \in(T S)\{1,2,4\}$. By using [1, Lemma 2.1.] we conclude that $T S$ has closed range and [15, Theorem 2.2] implies that $(T S)^{\dagger} T S=S^{\dagger} T^{\dagger} T S$.

(i) Since $S$ is EP operator with closed range, then by Lemma 1.4 operators $S$ and $T$ have the following matrix representations

$$
\begin{aligned}
& S=\left[\begin{array}{cc}
S_{1} & 0 \\
0 & 0
\end{array}\right]:\left[\begin{array}{l}
\operatorname{ran}(S) \\
\operatorname{ker}(S)
\end{array}\right] \rightarrow\left[\begin{array}{l}
\operatorname{ran}(S) \\
\operatorname{ker}(S)
\end{array}\right], \\
& T=\left[\begin{array}{ll}
T_{1} & T_{2} \\
T_{3} & T_{4}
\end{array}\right]:\left[\begin{array}{l}
\operatorname{ran}(S) \\
\operatorname{ker}(S)
\end{array}\right] \rightarrow\left[\begin{array}{l}
\operatorname{ran}(S) \\
\operatorname{ker}(S)
\end{array}\right] .
\end{aligned}
$$


Since $T S=S T^{*}$, then

$$
\left[\begin{array}{ll}
T_{1} & T_{2} \\
T_{3} & T_{4}
\end{array}\right]\left[\begin{array}{cc}
S_{1} & 0 \\
0 & 0
\end{array}\right]=\left[\begin{array}{cc}
S_{1} & 0 \\
0 & 0
\end{array}\right]\left[\begin{array}{cc}
T_{1}^{*} & T_{3}^{*} \\
T_{2}^{*} & T_{4}^{*}
\end{array}\right]
$$

or equivalently,

$$
\left[\begin{array}{ll}
T_{1} S_{1} & 0 \\
T_{3} S_{1} & 0
\end{array}\right]=\left[\begin{array}{cc}
S_{1} T_{1}^{*} & S_{1}^{*} T_{3}^{*} \\
0 & 0
\end{array}\right]
$$

By using (2.8) we have $T_{3} S_{1}=0$, therefore by the invertibility of $S_{1}$, we conclude that $T_{3}=0$. On the other hand, since $P_{\operatorname{ran}(\mathrm{S})} T=P_{\operatorname{ran}(\mathrm{S})} T P_{\operatorname{ran}(\mathrm{S})}$, then $P_{\operatorname{ran}(\mathrm{S})} T-P_{\operatorname{ran}(\mathrm{S})} T P_{\operatorname{ran}(\mathrm{S})}=P_{\operatorname{ran}(\mathrm{S})} T(1-$ $\left.P_{\text {ran }(\mathrm{S})}\right)=0$, that is, $T_{2}=0$. Hence

$$
T S=\left[\begin{array}{cc}
T_{1} & 0 \\
0 & T_{4}
\end{array}\right]\left[\begin{array}{cc}
S_{1} & 0 \\
0 & 0
\end{array}\right]=\left[\begin{array}{cc}
T_{1} S_{1} & 0 \\
0 & 0
\end{array}\right]
$$

By using a similar argument for the closedness of the range of $S_{1}$ and $S_{4}$ in this proof we imply that, since $T$ has closed range, then $T_{1}$ and $T_{4}$ have closed ranges and $T^{\dagger}=\left[\begin{array}{cc}T_{1}^{\dagger} & 0 \\ 0 & T_{4}^{\dagger}\end{array}\right]$. On the other hand, since $\left(T_{1} S_{1}\right) S_{1}^{-1} T_{1}^{\dagger}\left(T_{1} S_{1}\right)=T_{1} S_{1}$ and $S_{1}^{-1} T_{1}^{\dagger}\left(T_{1} S_{1}\right) S_{1}^{-1} T_{1}^{\dagger}=S_{1}^{-1} T_{1}^{\dagger}$ and $\left(\left(T_{1} S_{1}\right) S_{1}^{-1} T_{1}^{\dagger}\right)^{*}=\left(T_{1} T_{1}^{\dagger}\right)^{*}=T_{1} T_{1}^{\dagger}$, then $S^{\dagger} T^{\dagger} \in(T S)\{1,2,3\}$. Therefore, [15, Theorem 2.1] implies that $T S(T S)^{\dagger}=T S S^{\dagger} T^{\dagger}$.

(ii) Since $T S(T S)^{\dagger}=T S S^{\dagger} T^{\dagger}$ and $(T S)^{\dagger} T S=S^{\dagger} T^{\dagger} T S$, then by [15, Corollary 2.3] we have $(T S)^{\dagger}=S^{\dagger} T^{\dagger}$.

Theorem 2.2. Suppose that $\mathcal{X}$ is a Hilbert $\mathcal{A}$-module and $T, S \in \mathcal{L}(\mathcal{X})$ are EP operators with closed ranges and $T S=S^{*} T$. Then $T S$ has closed range and $T S(T S)^{\dagger}=T S S^{\dagger} T^{\dagger}$.

Moreover, if $P_{\operatorname{ran}(\mathrm{S})} T=P_{\operatorname{ran}(\mathrm{S})} T P_{\operatorname{ran}(\mathrm{S})}$, then

(i) $(T S)^{\dagger} T S=S^{\dagger} T^{\dagger} T S$;

(ii) $(T S)^{\dagger}=S^{\dagger} T^{\dagger}$.

Proof. Since $S$ is EP operator with closed range, then operators $T, S$ have the same matrix representations (2.6) and (2.7), respectively. Since $T S=S^{*} T$, then

$$
\left[\begin{array}{ll}
T_{1} & T_{2} \\
T_{3} & T_{4}
\end{array}\right]\left[\begin{array}{cc}
S_{1} & 0 \\
0 & 0
\end{array}\right]=\left[\begin{array}{cc}
S_{1}^{*} & 0 \\
0 & 0
\end{array}\right]\left[\begin{array}{cc}
T_{1} & T_{2} \\
T_{3} & T_{4}
\end{array}\right],
$$

or equivalently,

$$
\left[\begin{array}{ll}
T_{1} S_{1} & 0 \\
T_{3} S_{1} & 0
\end{array}\right]=\left[\begin{array}{cc}
S_{1}^{*} T_{1} & S_{1}^{*} T_{2} \\
0 & 0
\end{array}\right]
$$

Then $S_{1}^{*} T_{2}=T_{3} S_{1}=0$. By the invertibility of $S_{1}$ we conclude that $T_{2}=T_{3}=0$. Hence

$$
T S=\left[\begin{array}{cc}
T_{1} & 0 \\
0 & T_{4}
\end{array}\right]\left[\begin{array}{cc}
S_{1} & 0 \\
0 & 0
\end{array}\right]=\left[\begin{array}{cc}
T_{1} S_{1} & 0 \\
0 & 0
\end{array}\right] .
$$


A similar argument as in the proof of the previous theorem shows that we have

$$
S^{\dagger} T^{\dagger}=\left[\begin{array}{cc}
S_{1}^{-1} T_{1}^{\dagger} & 0 \\
0 & 0
\end{array}\right] \in(T S)\{1,2,3\}
$$

By using [1, Lemma 2.1], we conclude that $T S$ has closed range and [15, Theorem 2.1] implies that $T S(T S)^{\dagger}=T S S^{\dagger} T^{\dagger}$.

(i) Since $T$ is EP operator with closed range, therefore the operators $T$ and $S$ have the matrix representations (2.1) and (2.2), respectively. Since $T S=S^{*} T$, then

$$
\left[\begin{array}{cc}
T_{1} & 0 \\
0 & 0
\end{array}\right]\left[\begin{array}{ll}
S_{1} & S_{2} \\
S_{3} & S_{4}
\end{array}\right]=\left[\begin{array}{cc}
S_{1}^{*} & S_{3}^{*} \\
S_{2}^{*} & S_{4}^{*}
\end{array}\right]\left[\begin{array}{cc}
T_{1} & 0 \\
0 & 0
\end{array}\right]
$$

or equivalently,

$$
\left[\begin{array}{cc}
T_{1} S_{1} & T_{1} S_{2} \\
0 & 0
\end{array}\right]=\left[\begin{array}{cc}
S_{1}^{*} T_{1} & 0 \\
S_{2}^{*} T_{1}^{*} & 0
\end{array}\right]
$$

Hence $T_{1} S_{2}=0$, by invertibility of $T_{1}$ we conclude that $S_{2}=0$. On the other hand, since $S P_{\text {ran }}(T)=P_{\operatorname{ran}(\mathrm{T})} S P_{\text {ran }}(T)$, then $S_{3}=0$. Similar argument for (2.4) and (2.5) in the previous theorem, implies that $S^{\dagger} T^{\dagger}=\left[\begin{array}{cc}S_{1}^{\dagger} T_{1}^{-1} & 0 \\ 0 & 0\end{array}\right] \in(T S)\{1,2,4\}$. Therefore [15, Theorem 2.2] implies that $(T S)^{\dagger} T S=S^{\dagger} T^{\dagger} T S$.

(ii) Since $T S(T S)^{\dagger}=T S S^{\dagger} T^{\dagger}$ and $(T S)^{\dagger} T S=S^{\dagger} T^{\dagger} T S$, then by [15, Corollary 2.3] we have $(T S)^{\dagger}=S^{\dagger} T^{\dagger}$

Theorem 2.3. Suppose that $\mathcal{X}$ is a Hilbert $\mathcal{A}$-module and $T, S \in \mathcal{L}(\mathcal{X})$ are EP operators with closed ranges and $T S=S T^{*}=S^{*} T$. Then $T S$ has closed range and $(T S)^{\dagger}=S^{\dagger} T^{\dagger}$.

Proof. Since $T S=S T^{*}$, then Theorem 2.1 implies that $T S$ has closed range and $(T S)^{\dagger} T S=S^{\dagger} T^{\dagger} T S$. On the other hand, since $T S=S^{*} T$, then Theorem 2.2 shows that $T S(T S)^{\dagger}=T S S^{\dagger} T^{\dagger}$. Therefore [15. Corollary 2.3] implies that $(T S)^{\dagger}=S^{\dagger} T^{\dagger}$.

\section{References}

[1] T. Aghasizadeh, S. Hejazian, Maps preserving semi-Fredholm operators on Hilbert $C^{*}$-modules, J. Math. Anal. Appl., 354 (2009), 625-629. 2, 2

[2] R. Bouldin, The product of operators with closed range, Tôhoku Math. J., 25 (1973), 359-363. 1

[3] R. Bouldin, Closed range and relative regularity for products, J. Math. Anal. Appl., 61 (1977), $397-403$. 1

[4] D. S. Djordjević, Products of EP operators on Hilbert spaces, Proc. Amer. Math. Soc., 129 (2001), 1727-1731. 1

[5] D. S. Djordjević, Further results on the reverse order law for generalized inverses, SIAM J. Matrix Anal. Appl., 29 (2007), 1242-1246. 1

[6] M. Frank, Self-duality and $C^{*}$-reflexivity of Hilbert $C^{*}$-moduli, Z. Anal. Anwendungen, 9 (1990), 165-176. 1

[7] M. Frank, Geometrical aspects of Hilbert $C^{*}$-modules, Positivity, 3 (1999), 215-243. 1 
[8] T. N. E. Greville, Note on the generalized inverse of a matrix product, SIAM Rev., 8 (1966), 518-521. 1

[9] S. Izumino, The product of operators with closed range and an extension of the reverse order law, Tôhoku Math. J., 34 (1982), 43-52. 1

[10] E. C. Lance, Hilbert $C^{*}$-modules, A toolkit for operator algebraists, London Mathematical Society Lecture Note Series, Cambridge University Press, Cambridge, (1995). 1, 1.1

[11] M. Mohammadzadeh Karizaki, M. Hassani, M. Amyari, Moore-Penrose inverse of product operators in Hilbert $C^{*}$-modules, Filomat, (To appear). 1

[12] M. Mohammadzadeh Karizaki, M. Hassani, M. Amyari, M. Khosravi, Operator matrix of Moore-Penrose inverse operators on Hilbert $C^{*}$-modules, Colloq. Math., 140 (2015), 171-182 1

[13] K. Sharifi, The product of operators with closed range in Hilbert $C^{*}$-modules, Linear Algebra Appl., 435 (2011), 1122-1130. 1

[14] K. Sharifi, EP modular operators and their products, J. Math. Anal. Appl., 419 (2014), 870-877. 1.3. 1.4

[15] K. Sharifi, B. A. Bonakdar, The reverse order law for Moore-Penrose inverses of operators on Hilbert $\mathrm{C}^{*}$-modules, Bull. Iranian Math. Soc., 42 (2016), 53-60. 2, 2, 2, 2

[16] Q. Xu, L. Sheng, Positive semi-definite matrices of adjointable operators on Hilbert $C^{*}$-modules, Linear Algebra Appl., 428 (2008), 992-1000. 1 